\title{
Weak Lensing in Scalar-Tensor Theories of Gravity: Preliminary Results
}

\author{
Carlo Schimd \\ Institut d'Astrophysique de Paris, GReCO \\ 98 bis, bvd Arago, 75014 Paris, France \\ and \\ Dipartimento di Fisica, Università degli Studi di Parma \& INFN Gruppo Collegato di Parma \\ Parco Area delle Scienze, 7/A, 43100 Parma, Italy \\ email: schimd@iap.fr
}

\begin{abstract}
Scalar-tensor (ST) theories of gravity are the best motivated alternative to general relativity (GR), arising in every high-energy theory attempting to unify all the fundamental interactions. Furthermore, accomodating an arbitrary number of scalar fields, ST theories yield to cosmological scenarios with a dynamical realization of the dark energy. Solar-System experiments and binary-pulsars observations are compatible with very small departures from GR on the local universe $(z \simeq 0)$; on cosmological scales, big-bang nucleosynthesis and cosmic microwave background (CMB) observables can admit larger deviations from the predictions of GR. Weak lensing could provide a test for ST theories of gravity on intermediate scales. Based on a code developed to study ST theories on CMB observables, we implemented a plug-in code to compute the convergence power spectrum and some 2-points statistics. Preliminary results using a simple model of ST theory are presented. This study is aimed to constraint classes of ST models.
\end{abstract}

\section{Scalar-tensor theories of gravity: basics}

Scalar tensor theories of gravity are a natural alternative to general relativity, embedding it in a theoretical framework describing the gravitational interactions as mediated by the usual tensor field $g_{\mu \nu}$ (the metric) and by one or several scalar fields. Such theories are the most general description of the low-energy limit of superstring theories or, more generally, of those theories involving spatial extra-dimensions, where scalar degrees of freedom appear as physical fields (such as the dilaton or the moduli fields) or as mathematical representations of higher-order derivatives of the metric (for instance leading to $R^{2}, R$ being the Ricci scalar. See Lidsey, Wands \& Copeland (2000) for a review). Eventually, the additional scalar fields provide the common dynamical mechanism accounting for the primordial inflation and the actual accelerated expansion of the universe (Uzan (1999)).

To simplify we will consider ST models with a single scalar field $\varphi$ which strictly respect the weak equivalence principle (universality of free fall of laboratory-size objects). Using the signature $(-+++)$, the Lagrangian of the theory in the Jordan (i.e. physical) frame can be written as

$$
\mathcal{L}=\frac{1}{16 \pi G} F(\varphi) R-\frac{1}{2} \partial_{\mu} \varphi \partial^{\mu} \varphi-V(\varphi)+\mathcal{L}_{m}
$$

where $G$ is the bare gravitational constant and $\mathcal{L}_{m}$ the Lagrangian of the matter fields (dark matter and baryons). The Lagrangian (1.1) is completely defined by the coupling function $F(\varphi)$ and by the self-interacting potential $V(\varphi)$. GR corresponds to $F=1$ (referred as minimal coupling. With non-minimal coupling we refer to $F \neq$ constant); in this 
case the bare gravitational constant $G$ is the Newton constant. A flat potential $V$ without any dynamics of the scalar field leads to cosmologies with or without a cosmological constant $\Lambda$; "quintessence" scenarios are instead achieved through a non-trivial potential, like an inverse power law (the so-called Ratra-Peebles potential) with or without supergravity corrections (leading to the so-called SUGRA potentials).

The simplest ST model is described by a quadratic coupling, $F(\varphi)=1+8 \pi G \xi \varphi^{2}$. It is strongly constrained on the solar-system scales and on the Milky-Way scales $\left(\xi \varphi_{\text {today }} \lesssim\right.$ $10^{-3}$ ) by gravitational tests measuring the time delay of echo signals reflected by planets or spacecrafts or measuring the decay time of the orbiting period of binary-pulsars (see Bertotti, Iess \& Tortora (2003) for the most recent costraints). On cosmological scales, this model is moderately constrained at the epoch of the big-bang nucleosynthesis (BBN; $\left.\left|1-F\left(z_{\mathrm{BBN}}\right) / F(0)\right| \lesssim 20 \%\right)$, according to the observed abundances of the light elements (see Riazuelo \& Uzan (2002) and discussion therein).

Another interesting class of ST models firstly proposed by Damour \& Nordverdt (1993) regards the attractor mechanisms of the ST theories towards GR. Basically, for particular coupling functions $F(\varphi)$, as time goes on the Lagrangian (1.1) asymptotically reduces to that of GR. Contrary to the precedent class of ST models, this one is compatible with the small deviations from GR on the local universe $(z \simeq 0)$ according to the gravitational tests on the solar-system and binary-pulsars, but allows larger deviations from GR at high redshift.

\section{Gravitational lensing in ST theories of gravity}

To begin with, we examine a lens system in a static flat space-time, or equivalently at redshift $z=0$ (hence all quantities are denoted by a subscript 0 ). In the Einstein frame, related to the Jordan frame by a conformal transformation of the metric, $g_{\mu \nu}^{*}=F(\varphi) g_{\mu \nu}, \dagger$ the light deviation equation looks like the usual one formulated in GR,

$$
\Delta \theta=4 G M_{\mathrm{lens}, 0}^{*} / r_{0}^{*},
$$

where $\Delta \theta$ is the deviation angle of the light ray deflected by a lens with gravitational mass $M_{\text {lens }}^{*}$ and $r_{0}^{*}$ is the distance of closest approach to the lensing mass. Being the gravitational interactions mediated by the scalar field besides the usual graviton $g_{\mu \nu}^{*}$ (see figure 1), in ST theories one should define an effective gravitational constant,

$$
G_{\mathrm{eff}, 0}^{*}=G\left(1+\alpha_{0}^{2}\right)
$$

where $\alpha_{0}^{2}={F^{\prime}}^{2} /\left(3{F^{\prime}}^{2}+16 \pi G F\right)$ is the squared coupling constant of the scalar field in terms of the function $F$, a prime denoting the derivative with respect to $\varphi$. In fact, in the Jordan frame the effective gravitational constant measured by a Cavendish-type experiment writes

$$
G_{\mathrm{eff}, 0}=G_{\mathrm{eff}, 0}^{*} F^{-1}\left(\varphi_{0}\right) .
$$

Eventually, in the physical frame, the light deviation equation (2.1) becomes

$$
\Delta \theta=2\left(1+\gamma_{0}\right) G_{\mathrm{eff}, 0} M_{\mathrm{lens}, 0} / r_{0},
$$

$\gamma_{0} \equiv 2\left(1+\alpha_{0}^{2}\right)^{-1}$ being a post-Newtonian parameter measurable through gravitational experiments (the current constraint given by Bertotti, Iess \& Tortora (2003) is $\left|\gamma_{0}-1\right| \lesssim$ $\left.2 \times 10^{-5}\right)$. As for masses and distances, the Jordan and Einstein frames are related by $M_{\text {lens }, 0}=F^{1 / 2} M_{\text {lens }, 0}^{*}$ and $r_{0}=F^{-1 / 2} r_{0}^{*}$. Notice that these relations hold locally.

$\dagger$ All quantities defined in the Einstein frame are denoted by a superscript * . 


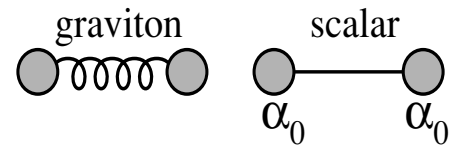

Figure 1. Diagrammatic representation of the gravitational interaction between two massive bodies (filled circles) in the Einstein frame, eq.(2.2).

The equation (2.4) is still valid for a lens system in an expanding universe, but geometrical and gravitational effects peculiar of ST theories are hidden. The background dynamics leads to a redshift dependence of coupling function $F$, modifying the redsfhift dependence of the (diameter) distance $r_{0}$ and driving a redshift dependence of the product $G_{\text {eff }} M_{\text {eff }}$. Simultaneously, the effective gravitational "constant" $G_{\text {eff }}$ is space-time dependent, because of the (a priori existing) subhorizon space-time fluctuations of the scalar field due to the non-minimal coupling. Thus the gravitational lensing - and specifically the weak lensing - could be an adequate tool to study the ST theories of gravity on cosmological scales, eventually at low redshift.

\section{Weak lensing: signatures of ST theories of gravity}

To evaluate and quantify the departures from GR, let's focus on the amplification matrix of a light bundle subtending an angle $\boldsymbol{\theta}$ by the observer. In a homogeneous and isotropic universe and in Born approximation it writes

$$
A_{a b}(\boldsymbol{\theta})=I_{a b}+\int_{0}^{\chi_{h}} d \chi g(\chi) S_{K}(\chi) \partial_{a b}^{2} \Phi\left(S_{K}(\chi) \boldsymbol{\theta}, \chi\right),
$$

$I_{a b}$ being the identity matrix $(a, b=1,2), \chi$ the comoving radial distance, $g(\chi)$ the usual lensing weight function accounting for the sources distribution as far as the horizon (at the comoving radial distance $\left.\chi_{h}\right), S_{K}(\chi)=(\sin \chi, \chi, \sinh \chi)$ the comoving diameter distance for $K=(1,0,-1)$ respectively and $\Phi \equiv \phi+\psi$ the deflecting potential in terms of the scalar perturbations of the metric $\phi, \psi \cdot \dagger$

Four generic effects specific to ST models will appear. We illustrate their amplitude on the simplest model of non-minimally coupled scalar field, $F(\varphi)=1+8 \pi G \xi \varphi^{2}$, and compare it to a $\Lambda$ CDM model.

i) Since the Hubble parameter $H(z)$ depends on $F(\varphi)$ and $V(\varphi)$, these functions modify the redshift dependence of the comoving radial distance $\chi=\int d z / H(z)$. With respect to the confidence $\Lambda$ CDM model, the difference can be of order $15-20 \%$ at redshift $z \simeq 2$ (see figure 2). In fact, accounting for the solar-system and binary-pulsars constraints on the ST model considered $\left(\xi \varphi_{\text {today }} \lesssim 10^{-3}\right)$, the more relevant variations are due to the self-interacting potential $V(\varphi)$.

ii) Owing to the non-minimal coupling and being $\phi-\psi=(d \ln F(\varphi) / d \varphi) \delta \varphi$, the deflecting potential is different from that computed in the GR framework $\left(\Phi_{\mathrm{GR}}=2 \phi\right)$. However, as far as the quadratic coupling function $F$ is concerned, according to Perrotta et al. (2004) the scalar field fluctuations $\delta \varphi$ cannot play any relevant role on sub-horizon scales. Therefore, at least for this model, the departures of the (power spectrum of the) deflecting potential from that computed in GR are completely negligible (see figure 3).

$\dagger$ The metric is defined by $d s^{2}=a^{2}(\eta)\left[-(1-2 \phi) d \eta^{2}+(1+2 \psi) d \mathbf{x}^{2}\right]$ using the conformal time $\eta$ and setting $c=1$. 


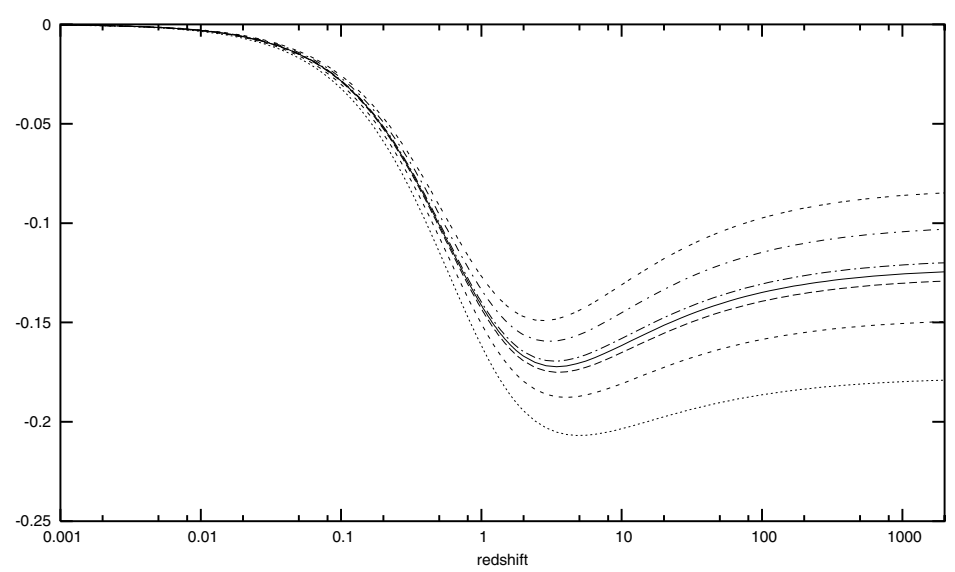

Figure 2. Relative deviation $\left(\chi / \chi^{\Lambda C D M}-1\right)$ from $\Lambda$ CDM on the comoving radial distance as a function of the redshift, for the model with $F(\varphi)=1+8 \pi G \xi \varphi^{2}$ and $V(\varphi)=\Lambda^{4+\alpha} \varphi^{-\alpha}$, $\alpha=6$. Below and above the solid line (minimal coupling, $\xi=0$ ), models with positive couplings ( $\xi=0.001,0.005,0.01$, from top to bottom) and negative couplings $(\xi=-0.001,-0.005,-0.01$, from bottom to top), respectively. Parameters of the model: flat universe, $\Omega_{\Lambda}=\Omega_{\varphi, 0}=0.7$, $h=0.72, \Omega_{b} h^{2}=0.019$.

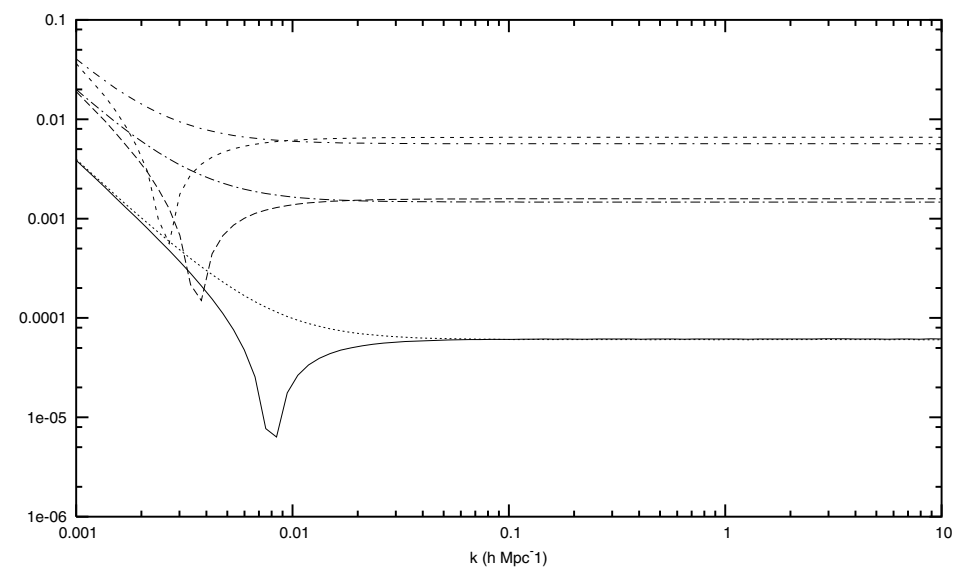

Figure 3. Relative deviation $\left|P_{\Phi} / P_{\Phi}^{\Lambda C D M}-1\right|$ from $\Lambda \mathrm{CDM}$ on the power spectrum of the deflecting potential $P_{\Phi}$, as a function of the wavevector $k$. Solid line: $\xi=+0.001$. Dotted line: $\xi=-0.001$. Long dashed line: $\xi=+0.005$. Long dotted-dashed line: $\xi=-0.005$. Short dashed line: $\xi=+0.01$. Short dotted-dashed line: $\xi=-0.01$. Parameters of the model as above.

iii) The Poisson and the Klein-Gordon equations are modified, essentially because of the redshift dependence of the Newton constant $G_{\text {eff }}$, eq.(2.3). See contribution of V. Acquaviva in these proceedings.

iv) Consistently with the precedent claim, the growth factor of the matter perturbations is changed with respect to that of the corresponding $\Lambda$ CDM model.

\section{Tool: the CMB-lensing code}

To study the effects of the ST theories of gravity on weak lensing observables, we implemented a plug-in code using the output of a Boltzmann code developed by Riazuelo \& Uzan (2002) to investigate the effects of such theories on CMB observables. The CMB 
code solves the Klein-Gordon and Einstein equations for cosmologies with a scalar field characterized by the self-interacting potential $V(\varphi)$ and the coupling function $F(\varphi)$. All the usual cosmologial parameters are taken into account and vector and tensor metric perturbations can be included as well. The output is the (linear) matter power spectra and the temperature and polarization power spectra at a desired redshift.

Once the CMB anisotropy spectrum is normalized on a single multipole or using a quadratic fit on low multipoles (Bunn \& White (1997)), the plug-in code computes the (linear) convergence power spectrum integrating the deflecting potential power spectrum along the line of sight, projected according the Limber equation and weighted by the function $g$ used in Van Waerbeke et al. (2001). Then it evaluates the aperture mass variance, the shear variance and the tangential and radial shear correlation functions.

To account for the non-linear clustering effects on the weak lensing observables, we compute the non-linear deflecting potential power spectrum using the non-linear Poisson equation, being negligible the difference between the scalar perturbations of the metric on the scales of interest at least (see ii) in Section 3). We apply the Smith et al. (2003) procedure to map the linear matter power spectrum into the non-linear one.

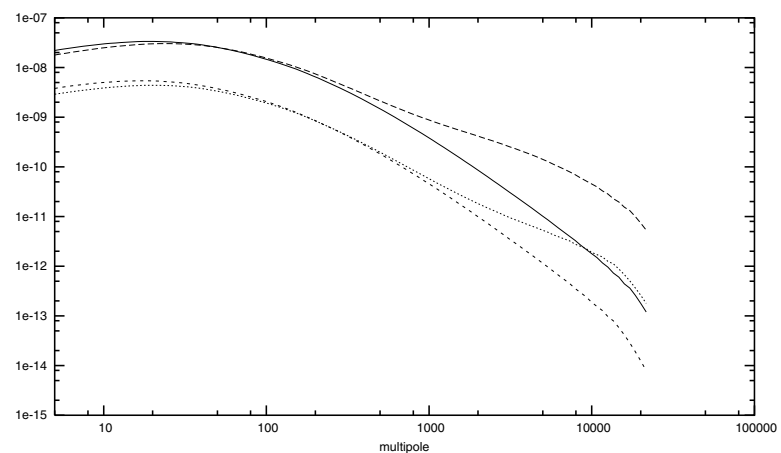

Figure 4. Convergence power spectrum $P_{\kappa}(\ell)$ as a function of the multipole $\ell$ for the $\Lambda$ CDM model and the non-minimally coupled model with $\alpha=6$. Solid (long dashed): linear (non-linear) regime for $\Lambda \mathrm{CDM}$. Short dashed (dotted): linear (non-linear) regime for non-minimally coupled model. Cosmological parameters as above.
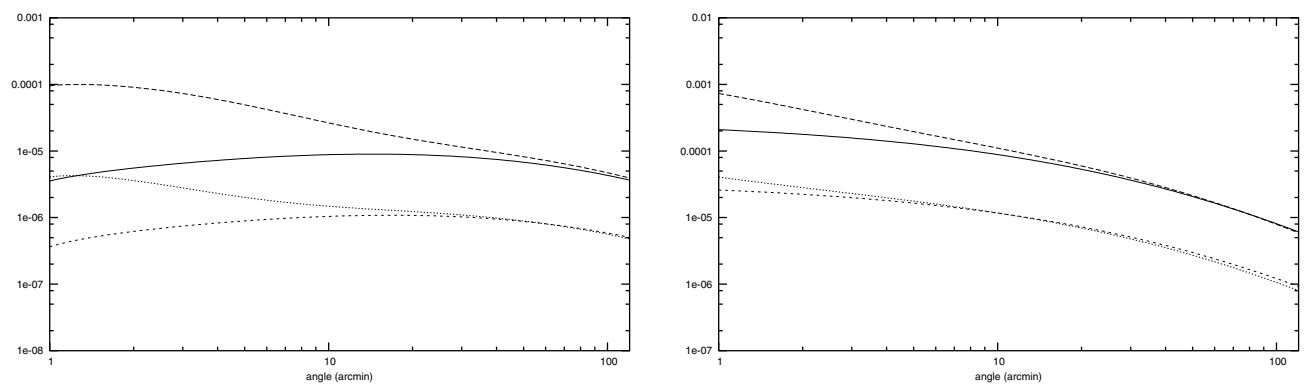

Figure 5. Aperture mass variance $\left\langle M_{a p}^{2}(\theta)\right\rangle$ (left) and shear variance $\left\langle\gamma^{2}(\theta)\right\rangle$ (right) as a function of the angle $\theta$ for the same models as in figure 4. Cosmological parameters as above.

Preliminary results are shown in figure 4 (convergence power spectrum) and figure 5 (aperture mass function and shear variance). The ST model considered is defined by an inverse power-law potential $V(\varphi)=\Lambda^{4+\alpha} \varphi^{-\alpha}, \alpha=6$ and by the quadratic coupling function $F=1+8 \pi G \xi \varphi^{2}$. The upper lines refer to the $\Lambda \mathrm{CDM}$ model with $\Omega_{\Lambda}=\Omega_{\varphi, 0}$, 
accounting for the linear and non-linear clustering effect respectively. The lower lines refer to the model with $\xi=0$; as expected, the departures from the minimally coupled model are negligible (below $1 \%$ ), thus not plotted. The deviations from the $\Lambda$ CDM curves (of order $10 \%$ ) are ultimately due to the non-flat potential $V(\varphi)$. Being normalized to WMAP, the variance $\sigma_{8}$ on a scale of $8 h^{-1} \mathrm{Mpc}$ does not agree with those inferred by galaxy clustering.

\section{Conclusions}

There are strong theoretical interests about ST theories of gravity, as providing the dynamical mechanism of the dark energy within the most general formulation of the lowenergy limit of the theories setting up spatial extra-dimentions. So far, all the departures from GR are quantified on the local universe $(z \simeq 0)$ and on the primordial universe, at the big-bang nucleosynthesis and at the photon-baryon decoupling era. We evaluate the ability of the weak lensing to constraint ST theories of gravity at low redshift, namely after the galaxy formation. The tool we implemented is a plug-in code based on a Boltzmann code, developed to study the effects on the CMB observables of cosmologies with a scalar field. Dealing with a Klein-Gordon equation, the scalar field provides a not parametrized realization of th dark energy. The output of the CMB code is used to compute the convergence power spectrum, the aperture mass variance, the shear variance and the shear correlation functions. All the results are normalized on the CMB anisotropy power spectrum at large angular scales.

Preliminary results on a simple ST model indicate that the most relevant signatures depend on the self-interacting potential of the scalar field, with negligible effects of its coupling to the metric. Thus weak lensing seems promising to constraint the "quintessence" potential. However, ST models may lead to more significant effects. A more detailed study of different potentials, as well as of more interesting couplings such as attractor mechanisms of ST towards GR, is in progress (see Schimd \& Uzan (2004)).

Nevertheless, such a study is meant to find out to which extent simulations and data analysis of weak lensing observables can be safely based on the standard GR framework, regardless the "true" underlying theory of gravitation.

\section{Acknowledgements}

I would thank Jean-Philippe Uzan, Gilles Esposito-Farèse and Yannick Mellier for fruitful discussions and encouragement, and Alain Riazuelo for kindly providing us his CMB code. Thanks to the organizers, the GReCO and the Marie-Curie programme "Improving the Human Research Potential and Socio-economic Knowledge Base" (contract number HMPT-CT-2000-00132) for the financial support.

\section{References}

Lidsey, E.L., Wands, D. \& Copeland, E.J. 2000, Phys. Rept. 337, 343-492.

Uzan, J.-P. 1999, Phys. Rev. D 59, 123510.

Bertotti, B., Iess, L. \& Tortora, P. 2003, Nature 425, 374.

Riazuelo, A. \& Uzan, J.-P. 2002, Phys. Rev. D 66, 023525.

Damour, T. \& Nordverdt, K. 1993, Phys. Rev. Lett. 70, 2217.

Perrotta, F., Matarrese, S., Pietroni., M. \& Schimd, C. 2004, Phys. Rev. D 69, 084004.

Bunn, E.F. \& White, M. 1997, ApJ 480, 6-21.

Van Waerbeke, L. et al. 2001, A\&BA 374, 757-769.

Smith, R. et al. 2003, MNRAS 341, 1311.

Schimd, C. \& Uzan, J.-P. 2004, to be submitted. 


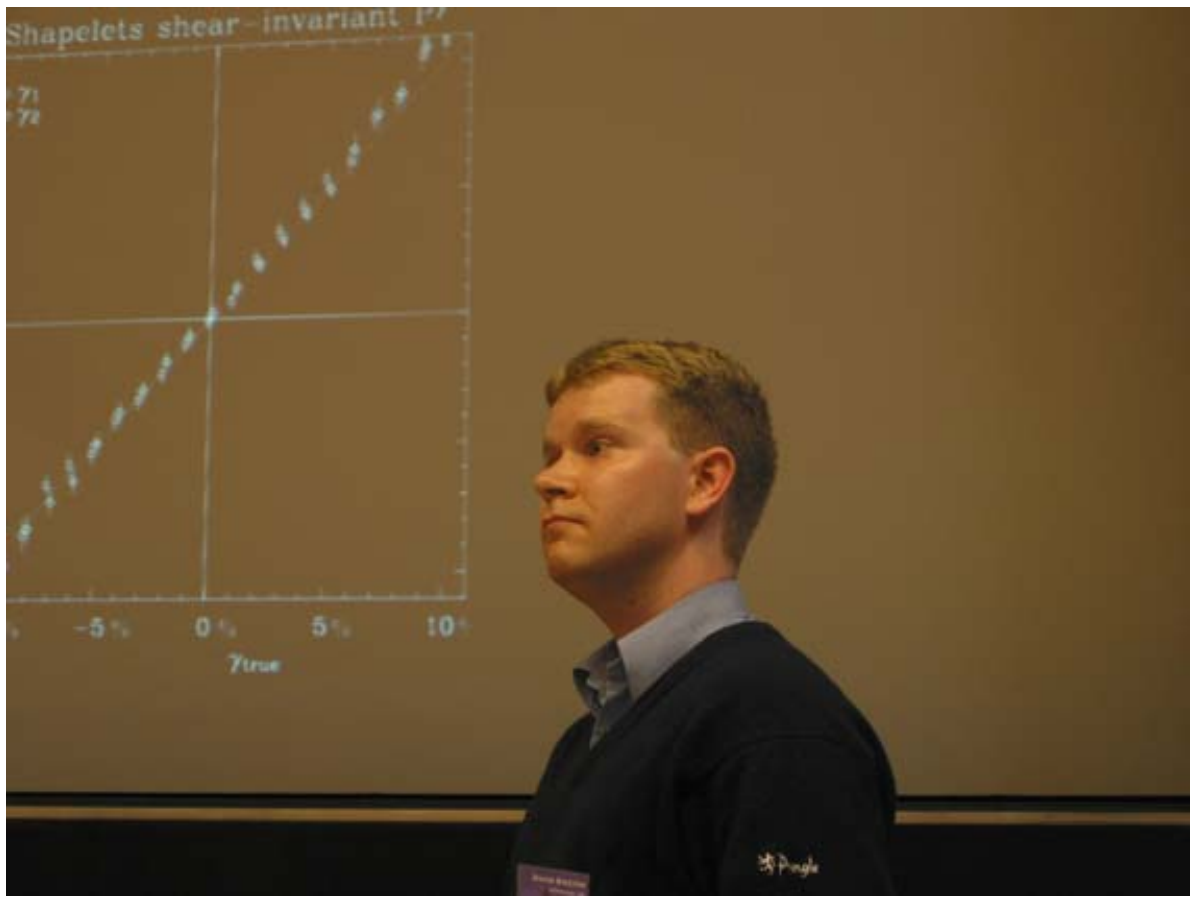

David J. Bacon

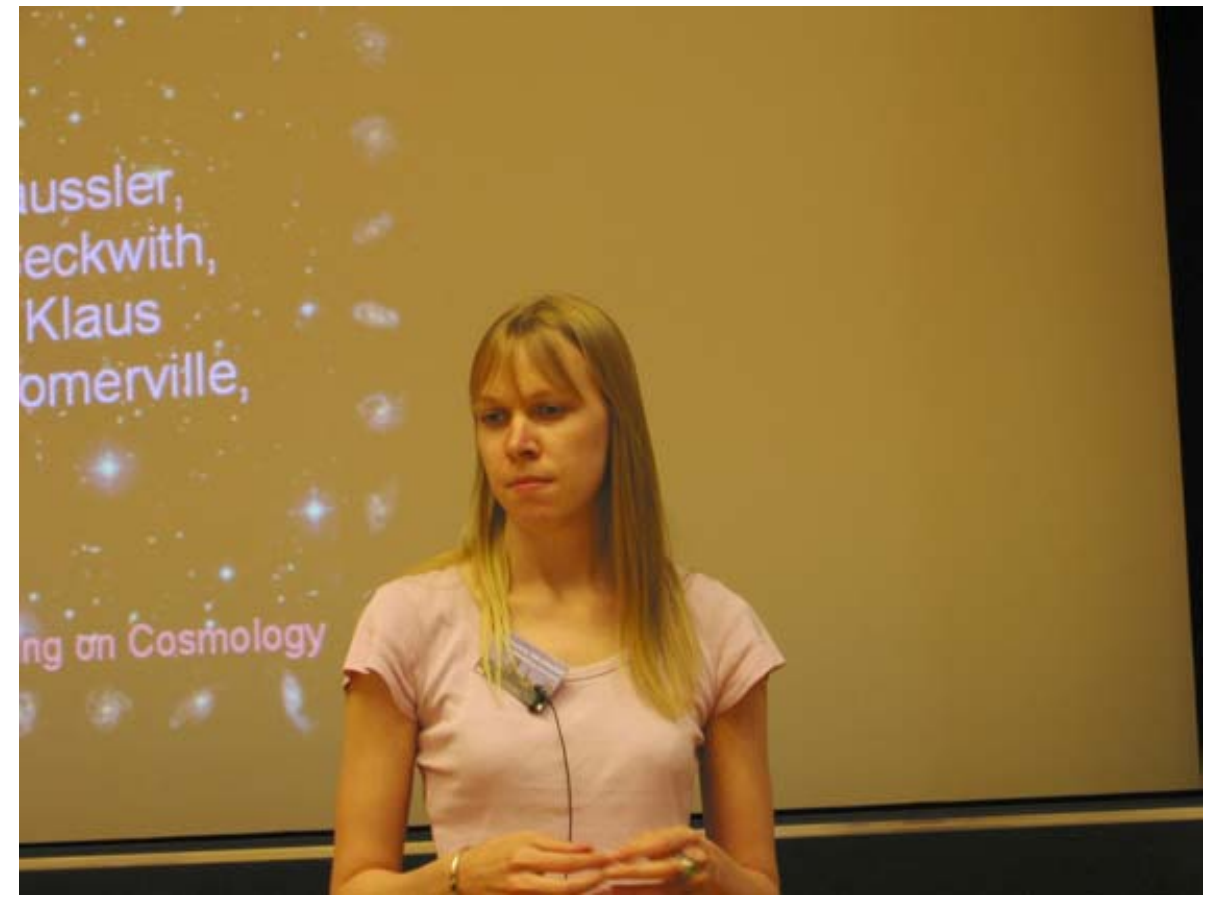

Catherine Heymans 

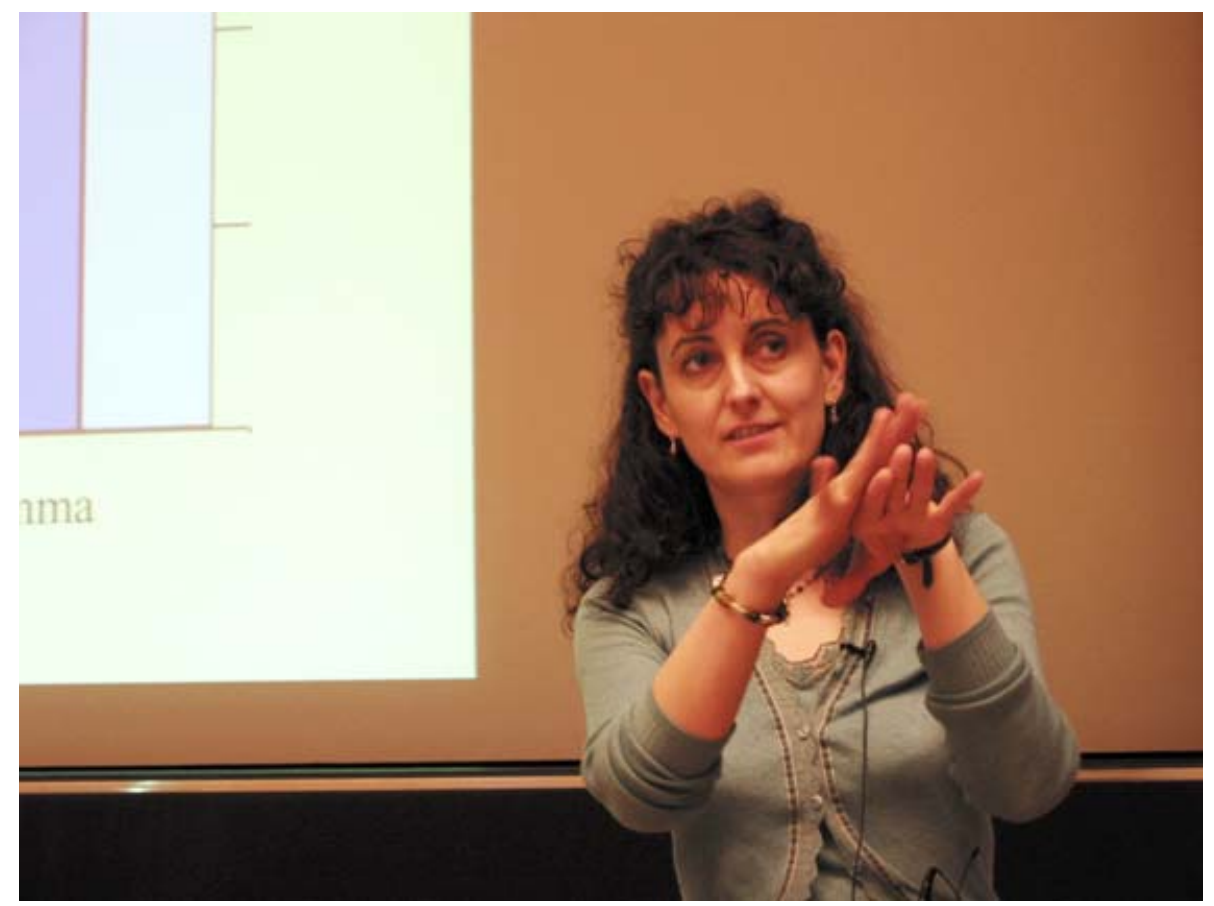

Lindsay J. King

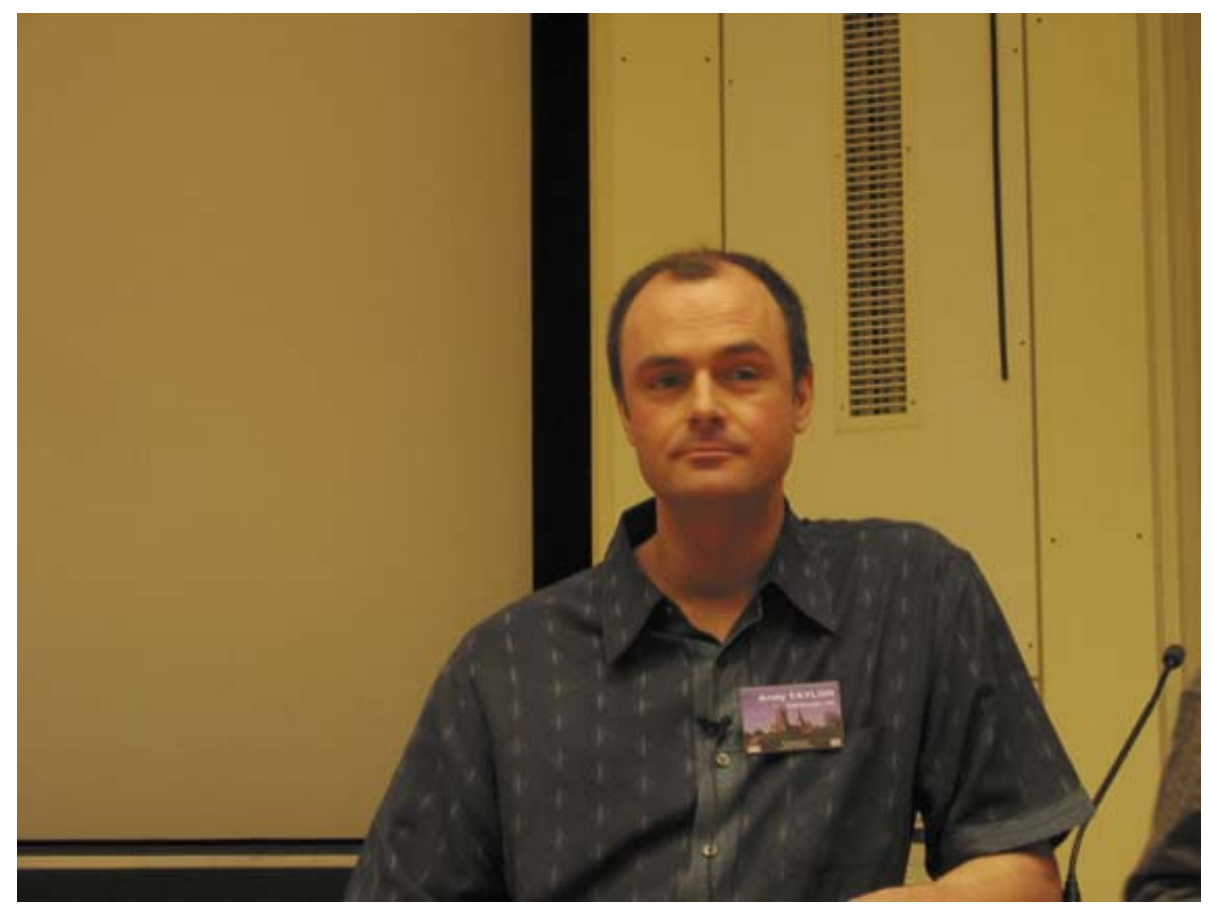

A. Taylor 


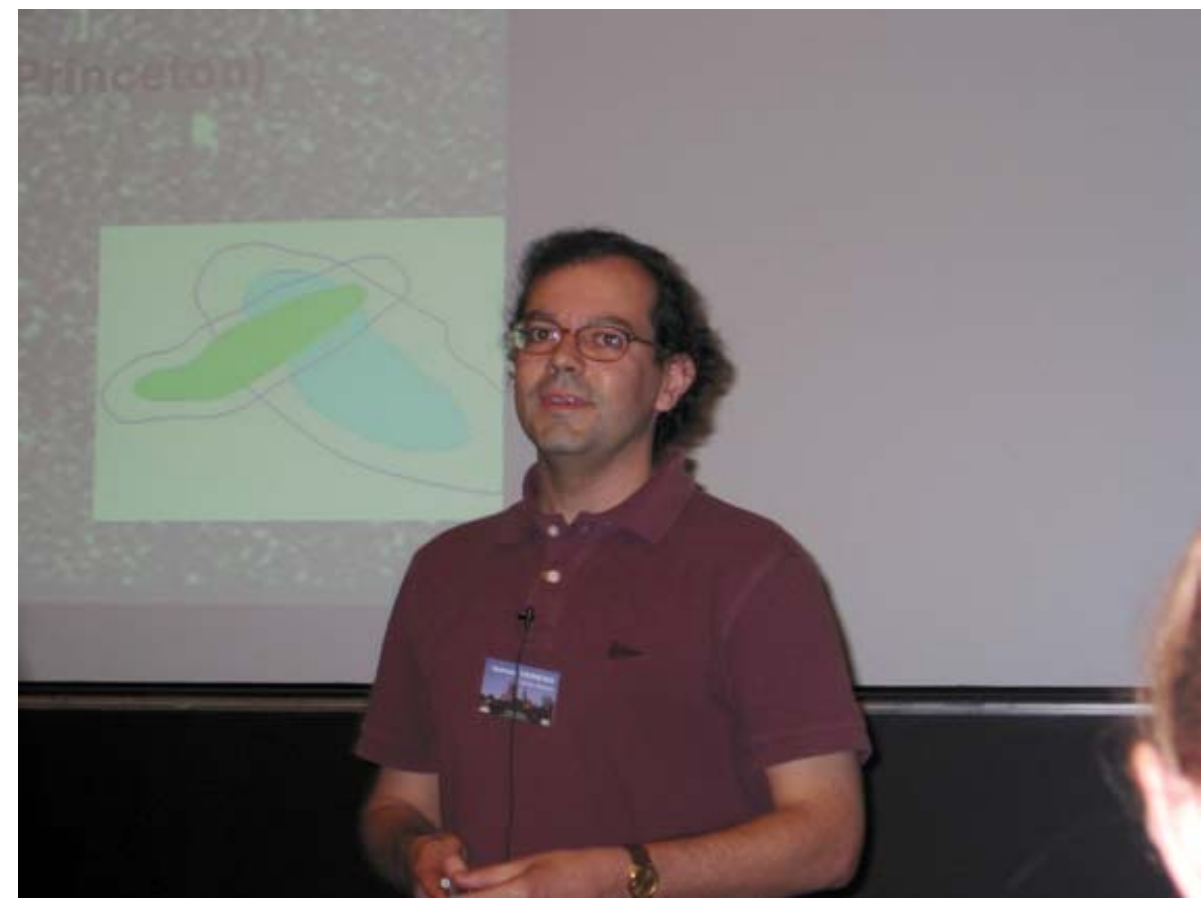

Ismael Tereno

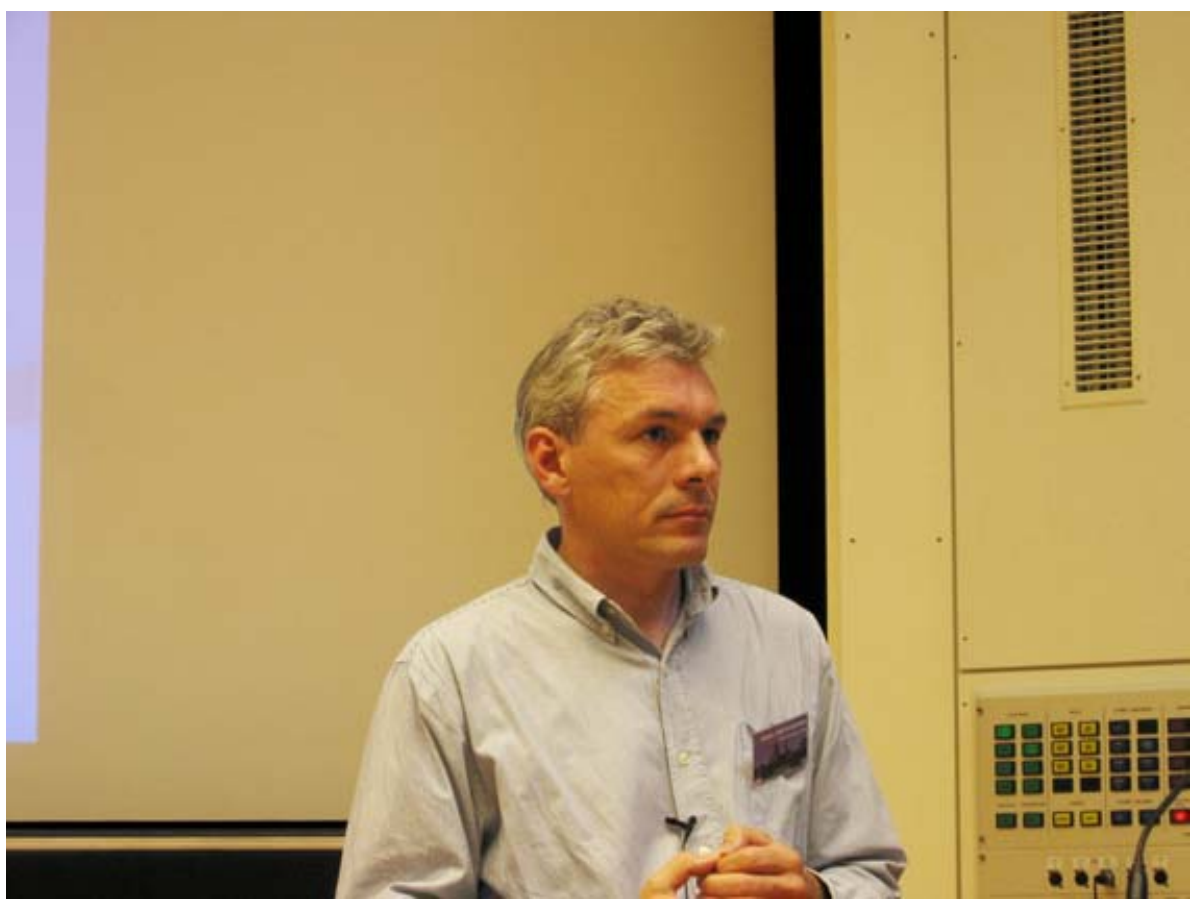

Alan F. Heavens 
ly to Torrioy! up itter distribution of n with cosmic shear

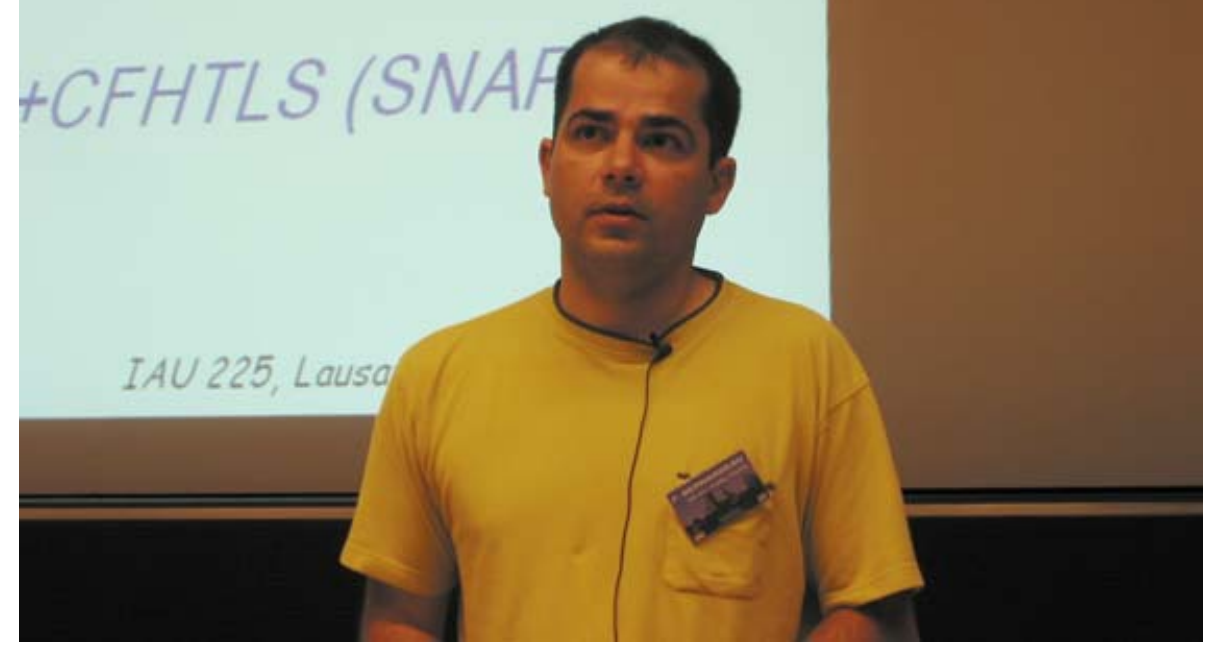

Francis Bernardeau

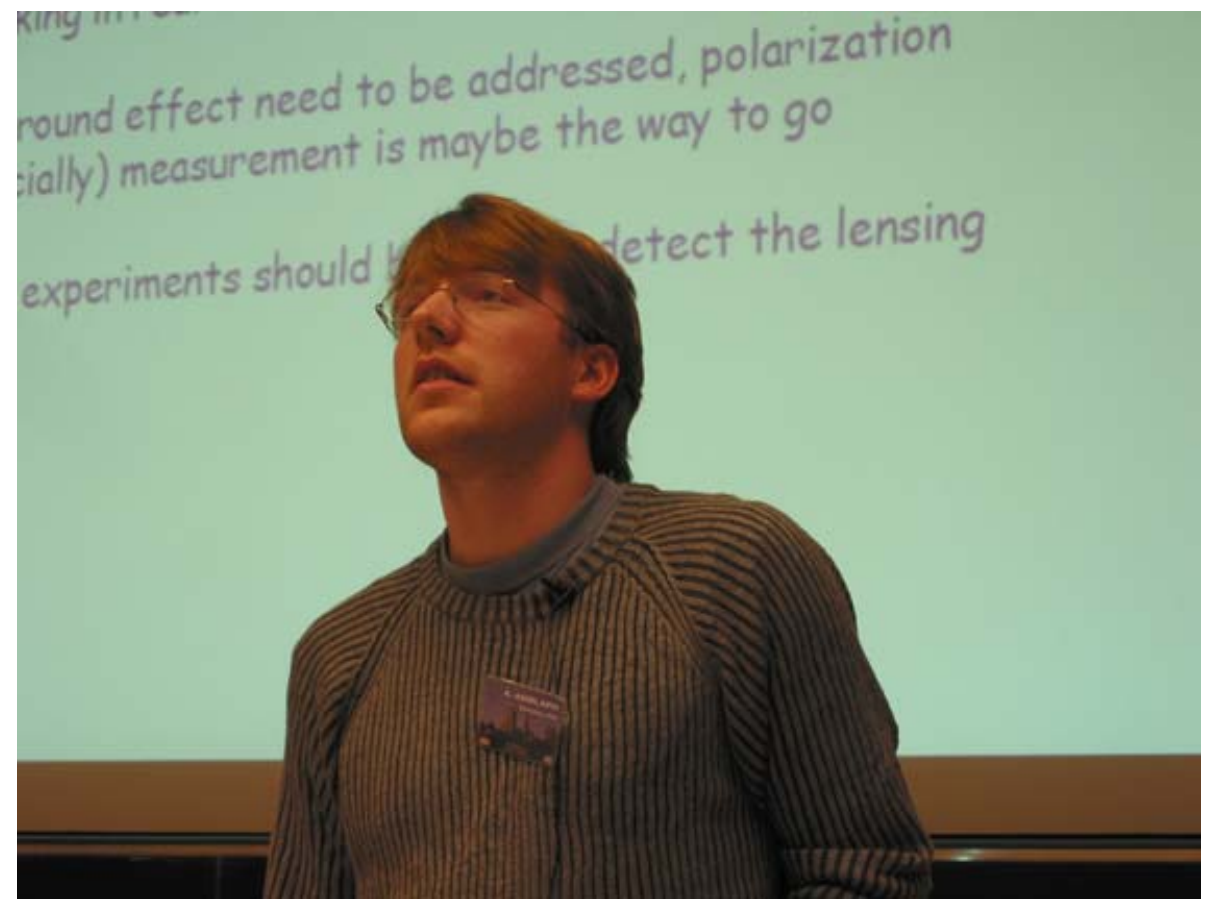

Alexandre Amblard 


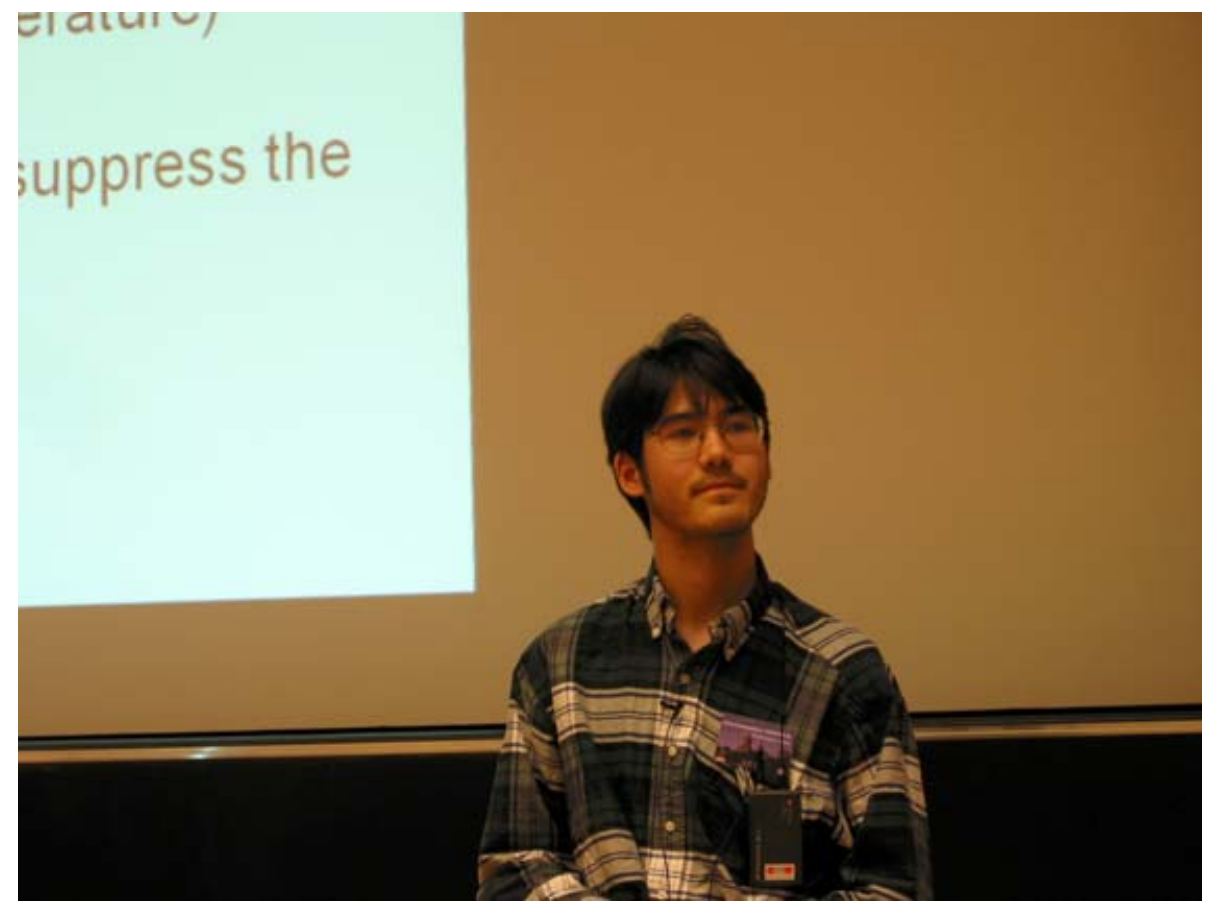

Christopher M. Hirata

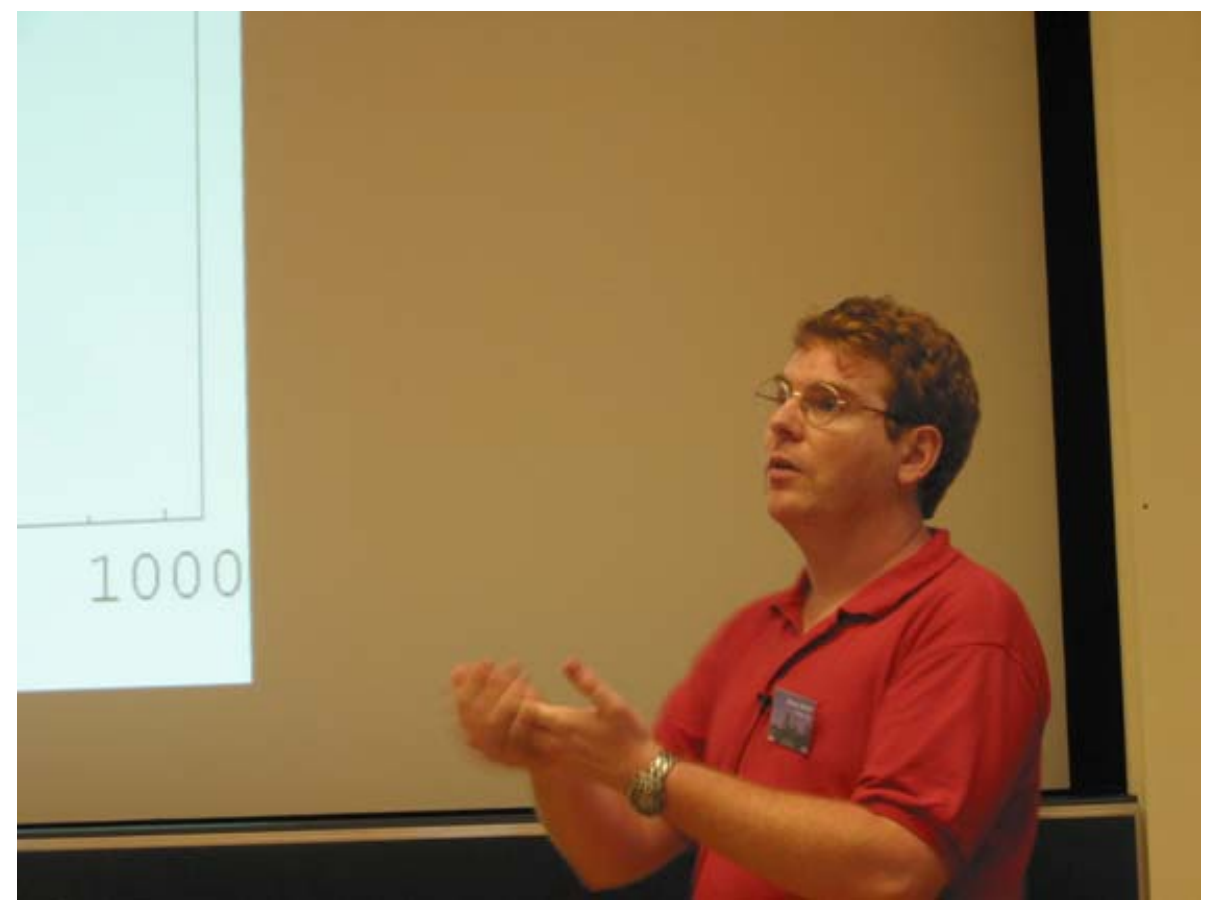

Fabio Giovi 